\title{
Fatigue Response of Solvent-Based Self-Healing Smart Materials
}

\author{
S. Neuser • V. Michaud
}

Received: 14 May 2013 / Accepted: 15 July 2013 / Published online: 13 August 2013

(C) Society for Experimental Mechanics 2013

\begin{abstract}
We investigated the healing of epoxy resins with embedded ethyl phenylacetate (EPA) solvent loaded capsules and shape memory alloy (SMA) wires under fatigue loading in tapered double cantilever beam (TDCB) mode. Under cyclic loading, the kinetics of solvent diffusion are in competition with the rate of damage propagation. We showed that the active mechanism of self-healing under continuous loading is different from that in quasi-static testing. Crack arrest was observed after some initial crack growth, resulting from the diffusion of EPA solvent into the crack tip, involving local plasticization of the epoxy. Finite element analysis confirmed that the lower modulus and higher elongation at break of the solvated epoxy reduced the stresses at the crack tip. This effect combined with the well-studied microcapsule toughening effect, tremendously increased the toughness of plain epoxy. For epoxy with embedded SMA wires, completely fractured samples, healed using SMA activation, showed similar fatigue resistance as virgin samples. Furthermore, SMA activation during a $10 \mathrm{~min}$ break also stabilized crack progression, compared to at least $2 \mathrm{~h}$ needed to reach the same effect without SMA wires.
\end{abstract}

Keywords Self-healing polymer · Smart composite · SMA $\cdot$ Fatigue testing

V. Michaud $(\bowtie) \cdot S$. Neuser

Laboratory of Polymer and Composite Technology (LTC),

Ecole Polytechnique Fédérale de Lausanne (EPFL),

CH-1015 Lausanne, Switzerland

e-mail: veronique.michaud@epfl.ch

\section{Introduction}

Polymers that can heal cracks after damage have been successfully demonstrated using different healing mechanisms [1-4]. Some systems are infinitely re-mendable and are based on reversible bond formation based on hydrogen bonding [5], ionomers [6] or reversible covalent bonding [7] and often require thermal or mechanical activation in order to heal damage. Other systems are completely autonomic, based on liquid healing agents either contained in microcapsules [8, 9], hollow glass fibers [10] or microvascular networks $[11,12]$ and can be healed a limited amount of times, depending on the availability of the healing agent.

Besides the systems mentioned above [13], advances in the field of smart materials have been made under the form of strain sensors, able to detect deformations and impacts $[14,15]$ and actuators used to locally provide heat and compressive forces to aid healing of damaged areas [16, 17]. These systems can be used to thermally trigger intrinsic selfhealing but are also appropriate for liquid-based self-healing by improving the healing agent mobility through increased diffusion or decreased crack opening [18]. While materials relying on an extrinsic liquid healing agent have an autonomous self-healing ability, it was shown that actuatoraided healing can improve healing efficiency by a factor 3 $[19,20]$.

In case of glassy structural polymers, specifically epoxide based resins, self-healing in static testing was demonstrated using 2-component healing agents (e.g. dicyclopentadiene (DCPD)/Grubbs catalyst [1], epoxy/mercaptan [21] or epoxy/amine [22]), as well as using 1-component solventfilled microcapsules [23]. During healing assessment, large 
damage is typically introduced into a sample under the form of cracks and after some healing time, in the order of the curing time of the healing agent, the restoration of mechanical properties is probed. In the context of solvent healing of an under-cured epoxy matrix, we showed in our previous work [20] that EPA solvent, upon release from the capsules, diffused into the resin following a Case II diffusion profile characterized by a sharp diffusion front $[24,25]$. The healing kinetics were closely linked to the diffusion rate of the solvent and corresponding swelling and it took $24 \mathrm{~h}$ to close and bond a $30 \mu \mathrm{m}$ crack gap through local matrix swelling. The saturation concentration of solvent was found to be $25 \%$ and the solvated epoxy showed important changes in mechanical properties as compared to plain epoxy: the Young's modulus was reduced from about 2.5 to $1.0 \mathrm{GPa}$ whereas the elongation at break increased almost threefold from 2.0 to $5.7 \%$.

Under continuous fatigue loading, the cure kinetics of the healing agent are in competition with the rate of damage propagation: if the crack propagation rate exceeds the rate of curing of the healing agent, minor effects as compared to non-self-healing samples are observed [26]. In contrast, if the damage rate is very small as compared to the healing rate or if sufficiently long rest periods are respected, the healing-agent is able to repair damage. Substantial life extension, expressed through increased cycle numbers to failure, was demonstrated and ranged up to $2000 \%$ for the DCPD/Grubbs catalyst system [27-29]. Beside the selfhealing effect, other factors contributing to fatigue life extension have been identified. Shin et al. reported that the formation of a solid wedge in the crack zone increased the unloading stress intensity $K_{\min }$ thereby lowering the stress intensity range $\Delta K$ [30]. Another effect is hydrodynamic shielding: if the crack tip is filled with liquid, viscous forces act on the crack surface, opposing crack closure and opening. Thereby the effective $\Delta K_{\text {eff }}$ is lower then the applied $\Delta K$ [27].

Self-healing with 1-component solvent microcapsules has been extensively investigated in static testing, while the performance in dynamic testing has only been briefly documented [31]. It was found that samples with solvent-filled microcapsules presented much slower crack growth rates as compared to control samples but the effect of rest periods and SMA wires has not yet been reported. Therefore in what follows, an epoxy matrix with a solvent-filled capsule healing system based on Caruso et al.'s work [23], combined with embedded shape memory alloy (SMA) fibers [20] is investigated under dynamic testing. We focus on the effect of solvent on the epoxy during fatigue testing, both for continuous experiments, as well as with breaks and SMA activation. Furthermore we investigate the compatibility of the solvent healing system with a fully cured epoxy matrix.

\section{Materials and Methods}

\section{Materials}

The epoxy resin was Epon 828, a DGEBA resin (Brenntag) which was cured with diethylenetriamine (DETA, Sigma Aldrich) in the stoichiometric ratio of 100:12. Curing took $24 \mathrm{~h}$ at ambient temperature followed by $24 \mathrm{~h}$ at $35^{\circ} \mathrm{C}$ (post-cure schedule 1, PC1), which led to an under-cured epoxy with a degree of cure of about $71 \%$ [32]. For fully cured samples (degree of cure $>95 \%$ ), a cycle of $24 \mathrm{~h}$ at ambient temperature followed by $6 \mathrm{~h}$ at $45^{\circ} \mathrm{C}$ and $45 \mathrm{~min}$ at $75{ }^{\circ} \mathrm{C}$ was used (post-cure schedule 2, PC2). Testing of samples was carried out 2 weeks after sample casting took place. A 97.5:2.5 mixture of ethyl phenylacetate (EPA $99 \%$, Sigma-Aldrich) and Epon 828 was used as the healing agent. Encapsulated hexyl acetate (Sigma-Aldrich) served as a control solvent with no healing ability [23]. Additionally, dicyclopentadiene (DCPD, Acros) combined with Grubbs' catalyst (Sigma Aldrich) was used for comparison. The SMA wire used in this study was a martensitic NiTiCu alloy with respective composition of 44.86/45.08/10.06 and a diameter of $150 \mu \mathrm{m}$ (Furukawa Techno Material). The phase transition temperatures as measured by differential scanning calorimetry (DSC) were $54.3{ }^{\circ} \mathrm{C}, 60.6{ }^{\circ} \mathrm{C}, 44.3^{\circ} \mathrm{C}$ and $37.3^{\circ} \mathrm{C}$ for $A_{s}, A_{f}, M_{s}$ and $M_{f}$, respectively.

\section{Encapsulation Procedure}

The EPA-Epon 828 microcapsules were produced using the "resin-solvent" urea-formaldehyde (UF) microencapsulation protocol established by Blaiszik et al. [33] using a stirring rate of $400 \mathrm{rpm}$. The DCPD capsules were produced using the "standard" UF encapsulation protocol [33] using a stirring rate of $550 \mathrm{rpm}$. In both cases, only the microcapsule fraction between 125 and $355 \mu$ m of diameter was retained using sieves. The EPA-Epon 828 microcapsules had an optically measured average diameter of $224 \pm 51 \mu \mathrm{m}$ and the DCPD microcapsules had an average diameter of $201 \pm 52 \mu \mathrm{m}$. The Grubbs' catalyst was recrystallized through dissolution in benzene followed by freeze-drying and wax protected as described by Jones et al. and Rule et al. respectively $[34,35]$.

\section{TDCB Sample Preparation}

Tapered double-cantilever beam (TDCB) samples, used both for fracture toughness measurement as well as for fatigue testing, were cast in silicone molds and left for curing for $24 \mathrm{~h}$. This geometry is characterized by a stress intensity $K_{I}$ which is linearly dependent on the applied load and independent of the crack length [36, 37]. Silicone spacers were used to minimize the amount of microcapsules per 
sample by casting in two steps: first the surrounding matrix consisting of pure epoxy resin, then the $15 \mathrm{wt} \%$ microcapsule loaded resin. For samples with integrated SMA wires, these were aligned using free-hanging $50 \mathrm{~g}$ weights attached to their ends before the silicone molds were closed, thereby clamping the SMA wires at 3 equally spaced locations as described in our previous work $[19,20]$. The samples were then stored at $21{ }^{\circ} \mathrm{C}$ and relative humidity of about $40 \%$.

\section{Fatigue Testing}

All fatigue testing was conducted on a Bose ElectroForce 3200 Series with a $225 \mathrm{~N}$ load cell. The TDCB epoxy samples were precracked by gently tapping a razor blade into the notch in order to produce a natural crack, then loaded into the testing frame. We applied a mode I tension-tension triangular wave form to the samples with an stress intensity ratio of $R=\frac{K_{\min }}{K_{\max }}=0.1$ and a frequency of $5 \mathrm{~Hz}$, based on the protocol defined by Brown et al. [27]. For pure epoxy matrix, samples were produced and aged for $0,1,5$, 14,21 and 35 days before a $\Delta K$ sweep $(0.37,0.39,0.42$ and $0.44 \mathrm{MPam}^{1 / 2}$ ) was performed in order to determine the Paris' law fitting parameters as a function of aging time. To do so, at a given stress intensity, the number of cycles necessary to advance the crack by $1 \mathrm{~mm}$ was used to compute the crack growth rate.

The crack position was visually determined with the help of a ruler parallel to the advancing crack which was held in place by an independent stand. In what follows, we estimated the crack length of TDCB samples based on an experiment where the visually determined crack length was linked to the measured sample compliance.

Furthermore, a control experiment was carried out to estimate the effect of manually injected EPA solvent on the sample compliance as the matrix weakening in the crack tip might lead to falsified crack position readings. Therefore samples with crack lengths of around $15-20 \mathrm{~mm}$ were loaded in tension to $40 \mathrm{~N}$ and the crack tip was manually filled with solvent. The crack was kept open in order to prevent any healing effect and the samples were than left for $24 \mathrm{~h}$, after which the compliance was again determined. As compared to a control experiment without the injected solvent, no differences were detected. For samples with embedded microcapsules, experiments with constant stress intensities $\Delta K$ (between 0.30 and $0.56 \mathrm{MPam}^{1 / 2}$ ) were performed until fracture. Besides continuously testing until fracture, we also investigated the effect of interrupting fatigue testing for varying amounts of time (for $10 \mathrm{~min}$, $2 \mathrm{~h}$ or $24 \mathrm{~h}$ ) to allow static healing. A low-cycle, high stress intensity test $\left(\Delta K=0.50 \mathrm{MPam}^{1 / 2}\right)$ was chosen in order to prevent crack arrest during continuous testing and single out the effect of the rest period duration. After 20000 cycles $( \pm 1 \mathrm{~h})$, a rest period was followed. Then another 15000 cycles were applied in order to determine the trend in crack progression after the rest period. For active healing in samples with integrated SMA wires, two types of tests were carried out: in a first series of tests, samples with embedded microcapsules and SMA wires were fractured in a quasi-static tensile mode, followed by SMA activation (an electrical current of $0.5 \mathrm{~A}$ per wire was applied) for $90 \mathrm{~min}$, as we showed that this leads to optimal healing [20]. These samples were then tested continuously in fatigue at different stress intensities ( 0.25 to $\left.0.54 \mathrm{MPam}^{1 / 2}\right)$. The second type of experiments on samples with microcapsules and SMA wires consisted in the same low-cycle, high stress intensity protocol with resting periods as described above except that the SMA wires were activated during the break for $90 \mathrm{~min}$ (for the shortest break, the activation was limited to $10 \mathrm{~min}$ ).

\section{Quasi-Static Healing}

Static healing performance was assessed through fracture toughness testing of TDCB samples loaded in tension at a displacement rate of $5 \mu \mathrm{m} / \mathrm{s}$ in a tensile testing load frame (UTS Testsysteme $\mathrm{GmbH}$ ). In detail, the pristine samples were precracked by tapping a razor blade into the molded starter-notch in order to introduce a natural crack, fractured, then left to heal for $24 \mathrm{~h}$ and tested again. The healing efficiency $\eta$ is derived from the ratio of the healed peak load $P_{h}$ over the virgin peak load $P_{v}$ [38].

An overview of all the experiments described in this work is found in Table 1.

\section{Fatigue Behavior of Pure Epoxy Matrix}

\section{Baseline Material Properties}

Before testing samples with embedded microcapsules and integrated SMA wires, baseline properties of the pure Epon 828 resin were retrieved. The parameters $C$ and $m$ of Paris' law (equation (1)) were obtained using a stress intensity sweep [39], for samples aged for different times.

$$
\frac{\mathrm{d} a}{\mathrm{~d} N}=C \Delta K^{m}
$$

Figure 1(a) plots the crack growth rate as a function of stress intensity per sample, each aged for a different duration of time. Evidently, high variability existed for samples tested less than 2 weeks after casting. The Paris's law exponent decreased with time as shown in Fig. 1(b), varying from 10.5 to 4.6 but stabilizing towards about 5.0 after 2 weeks. This stabilization over time could be due to minor water absorption (up to $0.3 \%$ after 2 weeks [32]), which has a plasticizing effect on the epoxy matrix [40]. 
Table 1 Experimental plan

\begin{tabular}{lllll}
\hline Capsules & Curing & SMA wires & Testing & $\Delta K\left[\mathrm{MPam}^{1 / 2}\right]$ \\
\hline- & PC1 & - & Continuous fatigue & $0.37-0.54$ \\
$-/$ DCPD/EPA & PC1 & - & Continuous fatigue & $0.30-0.56$ \\
EPA & PC1 & yes & Static followed by fatigue & $0.25-0.54$ \\
EPA & PC1 & yes & Fatigue +10 min, 2 h or 24 h breaks & 0.50 \\
EPA & PC3 & - & Static & - \\
EPA & PC3 & - & Continuous fatigue & $0.35-0.50$ \\
\hline
\end{tabular}

Therefore we left the samples rest for 2 weeks at $21^{\circ} \mathrm{C}$ and relative humidity of about $40 \%$ before fatigue testing for all subsequent testing to ensure a reproducible behavior. The Paris' law exponent reported here differed from the reported value of 9.7 by Brown et al. [29] but the difference

\section{(a)}

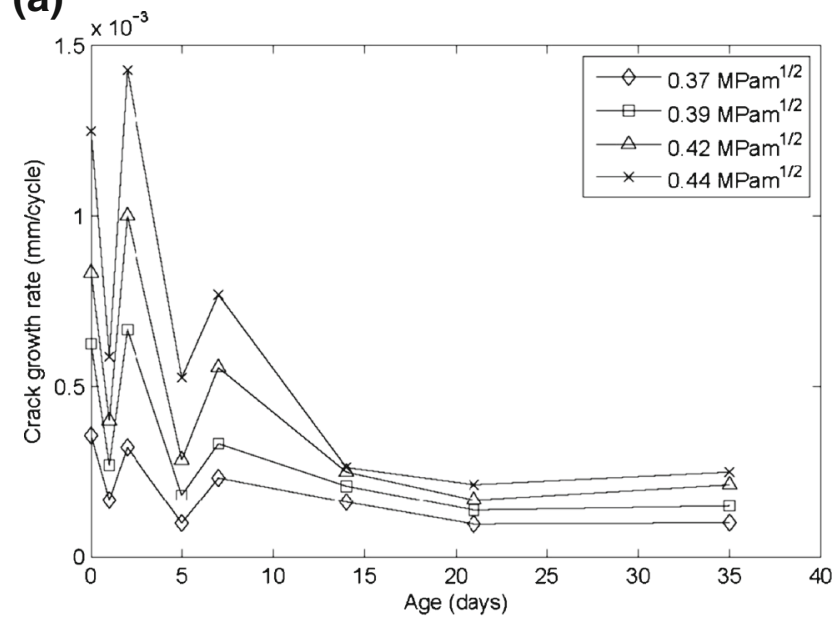

(b)

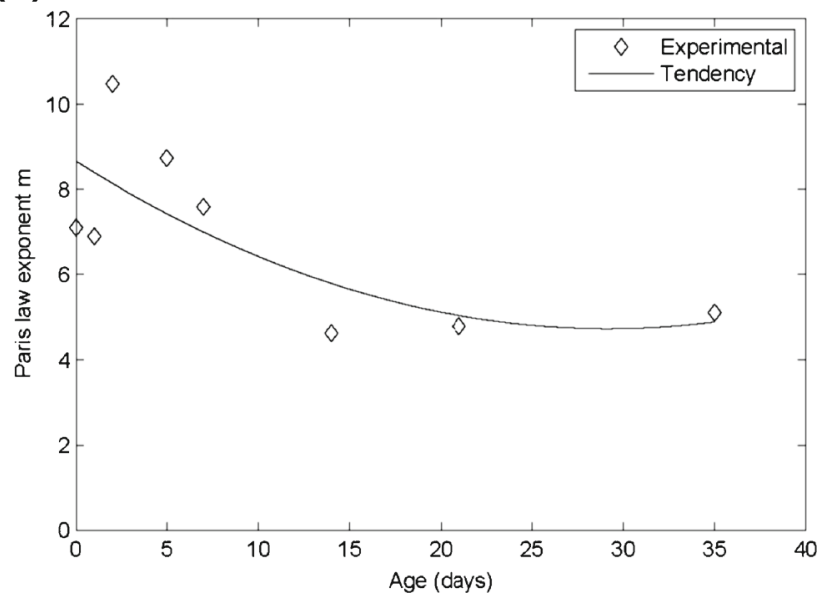

Paris'law exponent vs. aging

Fig. 1 Effect of aging on different material properties. (a) Crack growth rate as a function of aging time. (b) Paris' law exponent as a function of aging time can be attributed to the slight difference in PC temperature $\left(30{ }^{\circ} \mathrm{C}\right.$ vs. $35^{\circ} \mathrm{C}$ used in this work) and the waiting time of 2 weeks used in this study.

\section{Calibration Compliance/Crack Length}

Based on the above findings, 4 samples aged for 2 weeks were tested in fatigue at different stress intensities. The results, averaged over all 4 samples, are found in Fig. 2(a). As a result, for pure epoxy aged for 2 weeks, the averaged Paris' law components $C$ and $m$ were found to be $0.015 \frac{\mathrm{mm}}{{\text { cycle. } \mathrm{MPam}^{1 / 2}}^{1 / 2}}$ and 4.9 respectively. While for neat epoxy, the crack can be followed optically due to the transparent nature of the samples, this is not the case for the opaque epoxy samples loaded with microcapsules. Therefore a calibration curve relating sample compliance to crack progression was established and the result is given in Fig. 2(b). While the variability is not negligible, its effect is reduced to a shift in the ordinate of the conversion, which means that while the absolute value of crack location may be less reliable, the crack progression rate is quite accurate.

\section{Fatigue Behavior of Epoxy Matrix with Embedded Self-healing Agent}

Next, first fatigue tests on plain and microcapsule loaded TDCB samples were performed according to the protocol detailed above. The samples were tested continuously without interruption. In the first instance, we specifically compared plain epoxy to the well-known DCPD/Grubbs self-healing epoxy and the EPA solvent-loaded epoxy. Representative results are presented in Fig. 3. At constant $\Delta K=0.47 \mathrm{MPam}^{1 / 2}$ loading, a crack in the plain sample progressed rapidly while in the DCPD/Grubbs sample progression was slower, in accordance with Brown et al. [29]. EPA solvent microcapsule loaded TDCB samples also showed a reduced crack progression rate as compared to the plain epoxy, but after about 72000 cycles $( \pm 4 \mathrm{~h})$ for this sample, complete crack arrest was observed at these relatively high loading conditions.

Figure 4 shows crack propagation as a function of different stress intensities, based on the same EPA solvent 
(a)

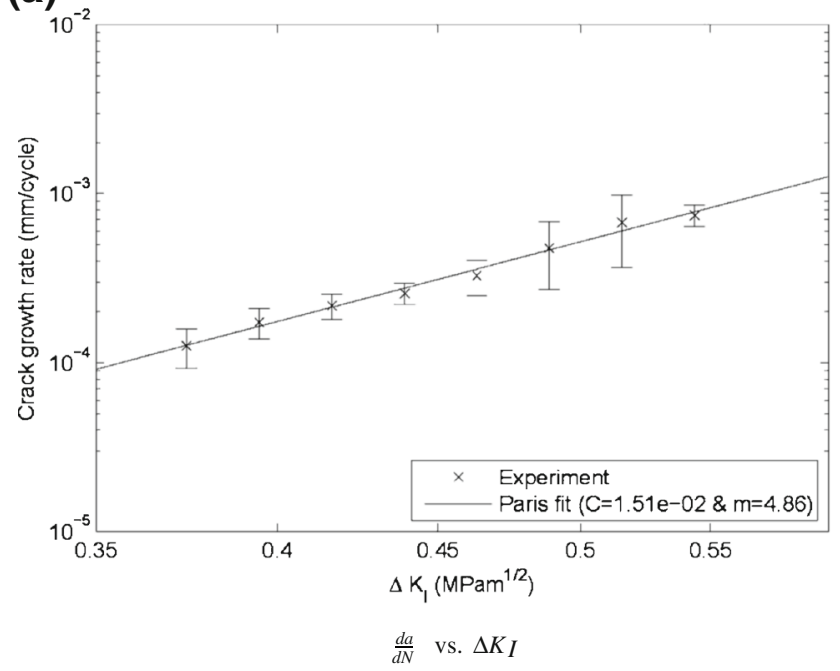

(b)

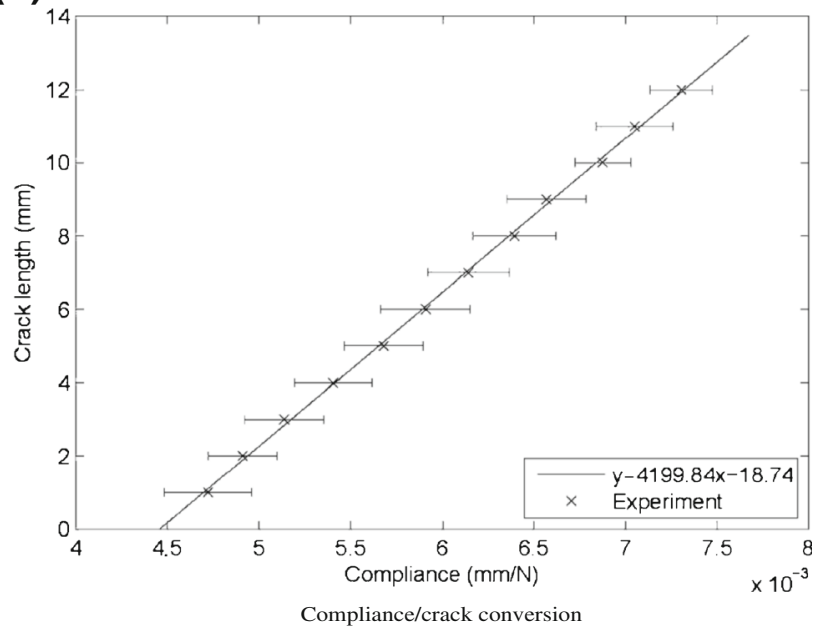

Fig. 2 Calibration data for the Epon828/DETA polymer. (a) Paris' law fitting at different stress intensities with error bars based on 3 to 4 measurements. All samples were tested at least 2 weeks after processing. (b) Conversion of compliance into optically measured crack length on pure epoxy samples

microcapsule loaded TDCB samples. For comparison, we also plot the results for the control samples with embedded hexyl acetate microcapsules. In case of EPA solvent and for low loading conditions $\left(\Delta K=0.3\right.$ and $0.41 \mathrm{MPam}^{1 / 2}$ ), no crack progression took place while for higher load conditions (above $\Delta K=0.48 \mathrm{MPam}^{1 / 2}$ ), the crack progressed for about $5 \mathrm{~mm}$ after 200000 cycles $( \pm 11 \mathrm{~h})$, before reaching a rather constant value. For $\Delta K=0.56 \mathrm{M} \mathrm{Pam}^{1 / 2}$, crack progression was fast and followed the same trend as for plain epoxy samples. This observation is in agreement with the findings of Caruso et al. [31]. At the same time, control samples with hexyl acetate filled microcapsules showed crack progression at much lower stress intensities.

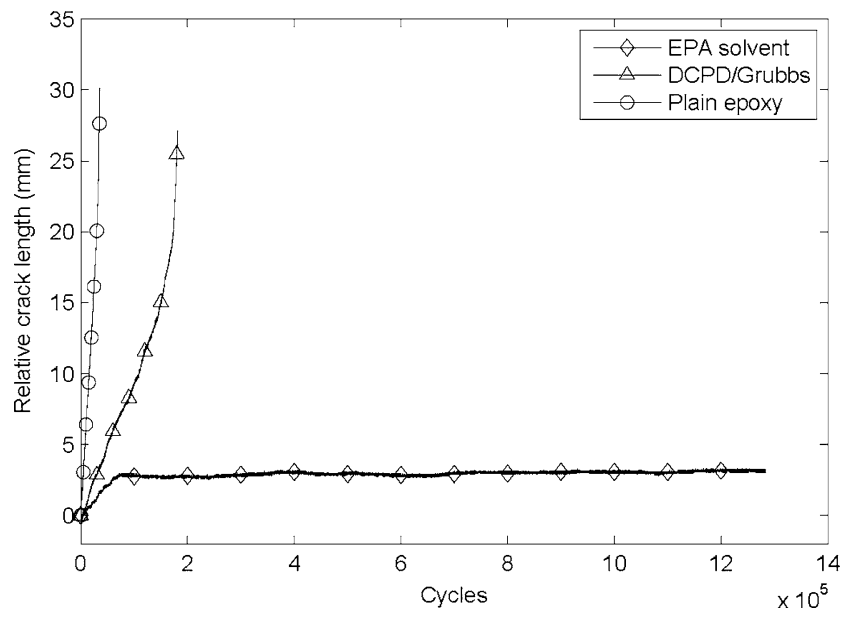

Fig. 3 Comparison between plain and microcapsule loaded TDCB samples without SMA wires fatigued at a constant $\Delta K=$ $0.47 \mathrm{MPam}^{1 / 2}$. A crack in the plain sample progresses rapidly while in the DCPD/Grubbs sample crack progression is slower. In the EPA solvent microcapsule loaded TDCB sample, crack arrest occurred after 7200 cycles

$\Delta K=0.41 \mathrm{MPam}^{1 / 2}$ already led to failure after about 100000 cycles.

As the samples were tested continuously with no rest time to heal, crack arrest cannot be due to mending of cracked surfaces as is the case in quasi-static tensile testing where the samples are completely broken, put together and left $24 \mathrm{~h}$ at room temperature in order to allow the formation of new crosslinked polymeric material. But neither is it caused by solid wedge formation under cyclic loading as reported for the DCPD/Grubbs healing system as solvent healing is based on the reaction of residual monomer rather than forming a new adhesive phase. The only active

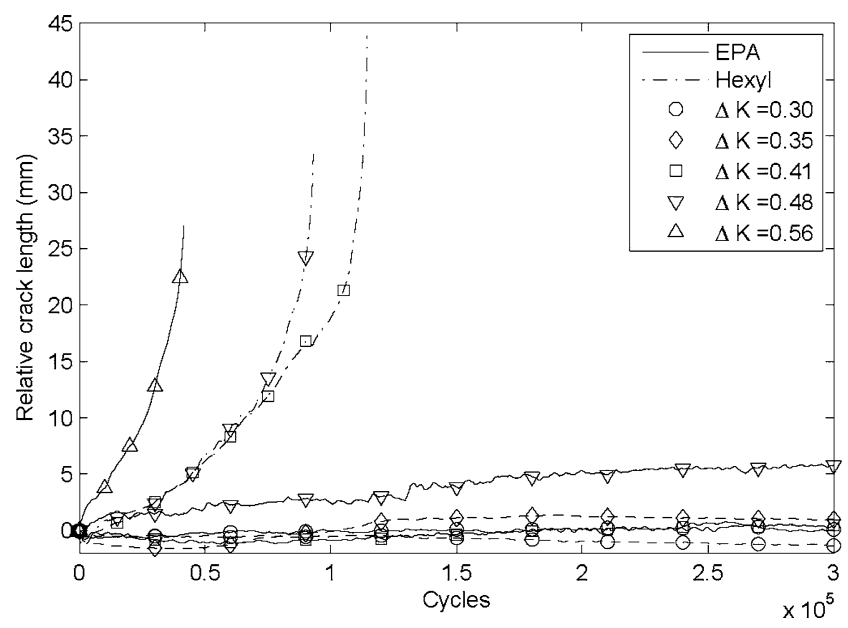

Fig. 4 TDCB samples loaded with 15 wt $\%$ EPA or hexyl acetate (control) microcapsules without SMA wires tested at different stress intensities. At stress intensity levels of $\Delta K=0.41$ and $0.48 \mathrm{MPam}^{1 / 2}$, failure occurred in the hexyl acetate control samples while EPA samples only failed at stress intensities well above $\Delta K=0.48 \mathrm{MPam}^{1 / 2}$ 
mechanisms in our case are the toughening effect due to the incorporation of microcapsules and the hydrodynamic pressure crack-tip shielding due to the presence of the solvent in the crack tip [41]. Therefore the difference between the control solvent and EPA must be due to solvent diffusion in the matrix material close to the crack tip and this effect will be investigated in the next section.

\section{FEA Modeling of Crack Tip}

Based on the experimentally measured diffusion kinetics [20], one can predict the swelling thickness from solvent diffusion as a function of time. Through interpolation, we found that it takes about $2 \mathrm{~h}$ for a solvent uptake equivalent to a swelling of $1 \mu \mathrm{m}$. This duration will be used as a representative time it takes for crack arrest to occur (equaling 36000 cycles). Since the saturation concentration is $25 \mathrm{wt} \%$, the penetration depth of the diffusion front is calculated to be around $4 \mu \mathrm{m}$. Based on these values, we investigate the solvent effect on stress and strain distribution around the crack tip using the finite element method (FEM). The analysis was carried out using ANSYS (ANSYS Inc.) [42] and a 2D crack tip model was generated. Figure 5(a) shows the chosen geometry and the boundary conditions which were used for this model. Using the symmetry of this problem, the bottom boundary is clamped while a tensile stress of $1 \mathrm{MPa}$ is applied to the top boundary. The crack tip notch protrudes by $100 \mu \mathrm{m}$ into the $400 \mu \mathrm{m}$ wide geometry. Here the crack tip was modeled by a simplified circular notch of $1 \mu \mathrm{m}$ of diameter, surrounded by the solventweakened layer of $4 \mu \mathrm{m}$ thickness. This numerical model consisted of 14051 nodes/4459 elements and isotropic elasticity and plane strain were used as the feature size is much smaller than the sample thickness. Finally we are interested in the stress and strain distribution along the path defined in the Fig. 5(b).

We then solved the problem both for pure epoxy with a Young's modulus of $2.5 \mathrm{GPa}$ as well as for epoxy with a solvent-affected epoxy zone, with a reduced Young's modulus of 1.0 GPa, based on our previous findings [20]. Color mapped equivalent von Mises stress plots for both cases are found in Fig. 6. The stress distribution is clearly affected by the sudden increase in Young's modulus when going from the crack tip towards the bulk.

In detail, the strains and stresses along the path defined above were extracted and are plotted in Fig. 7 for pure epoxy as well as the epoxy/solvent. The maximal strain found at the crack tip increased slightly from $1.50 \times 10^{-2}$ to $2.18 \times 10^{-2}$ due to the lower Young's modulus in the epoxy/solvent layer. Meanwhile, the maximal stresses at the crack tip were significantly reduced, from $38.1 \mathrm{MPa}$ to $21.8 \mathrm{MPa}$. While strain showed a continuous evolution, apart from a small step in slope at $4 \mu \mathrm{m}$ (the transition between epoxy and epoxy/solvent), stress first decreased until the material transition where it jumped back from 1.54 MPa to 4.69 MPa and then decreased again. At a distance around $2 \times$ the thickness of the epoxy/solvent layer, stresses and strains converged to the same value. In summary, we found that maximal strains increased by about $45 \%$ while the maximal stress decreased by $43 \%$ with the appearance of a second local maximum. While in reality the transition is less sharp and there probably exists a transition zone, even if very thin, the trends shown here clearly indicate that the solvent lowers the crack tip stresses through a reduced modulus, similar in effect to crack blunting. This phenomenon combined with the increased elongation at break therefore effectively stops crack propagation. Thus the initially observed high crack propagation rate preceding crack stabilization, is due to the time it takes for the solvent diffusion to saturate a sufficiently thick zone around the crack tip.

\section{Epoxy with Self-Healing Agent and SMA Wires}

\section{Fatigue on SMA Healed Samples}

While we showed that solvent diffusion is responsible for crack arrest under continuous fatigue testing, we also considered the case of a completely fractured sample using quasi-static fracture toughness testing, followed by a rest period to allow crack face bonding, in order to assess the fatigue resistance of the healed matrix. In our previous work, we showed that healing efficiency in quasi-static fracture toughness testing for solvent based self-healing epoxy can be as high as $80 \%$ if SMA wires are used in an optimized way [20]. As this value is directly dependent on the fracture toughness of the healed material, as is crack growth rate, we expect fatigue resistance to vary accordingly.

Therefore, after quasi-static fracture and healing through 90 min activation of the SMA wires followed by $24 \mathrm{~h}$ at room temperature, we subjected TDCB samples to different $\Delta \mathrm{K}$ loading conditions under fatigue. We also tested unfractured virgin TDCB samples with the same activation time of the SMA wires as a control group. Results are found in Fig. 8. Unsurprisingly, the virgin samples, where the SMA wires were activated prior the fatigue testing, resisted very well to the chosen loading conditions. As seen beforehand, samples without SMA wires failed eventually at loads above $\Delta K=0.50 \mathrm{MPam}^{1 / 2}$. Here, samples with SMA wires, activated for $90 \mathrm{~min}$, did not show any crack progression even at $\Delta K=0.54 \mathrm{MPam}^{1 / 2}$. Note that a slight "negative" crack propagation was often observed, in the order of $1 \mathrm{~mm}$, and we attribute this effect to a slight signal drift in the load cell. 
Fig. 5 (a) Geometry and boundary conditions of the crack tip model. A stress of $1 \mathrm{MPa}$ is applied to the top boundary while the bottom boundary is clamped. The crack tip notch protrudes by $1 / 4$ of the width of the geometry. (b) Detailed representation of the crack tip showing the two material regions. The inner, $4 \mu \mathrm{m}$ wide region, has a lower modulus as compared to the bulk. Stress and strain will be computed along the path between location 1 and 2

\section{(a)}

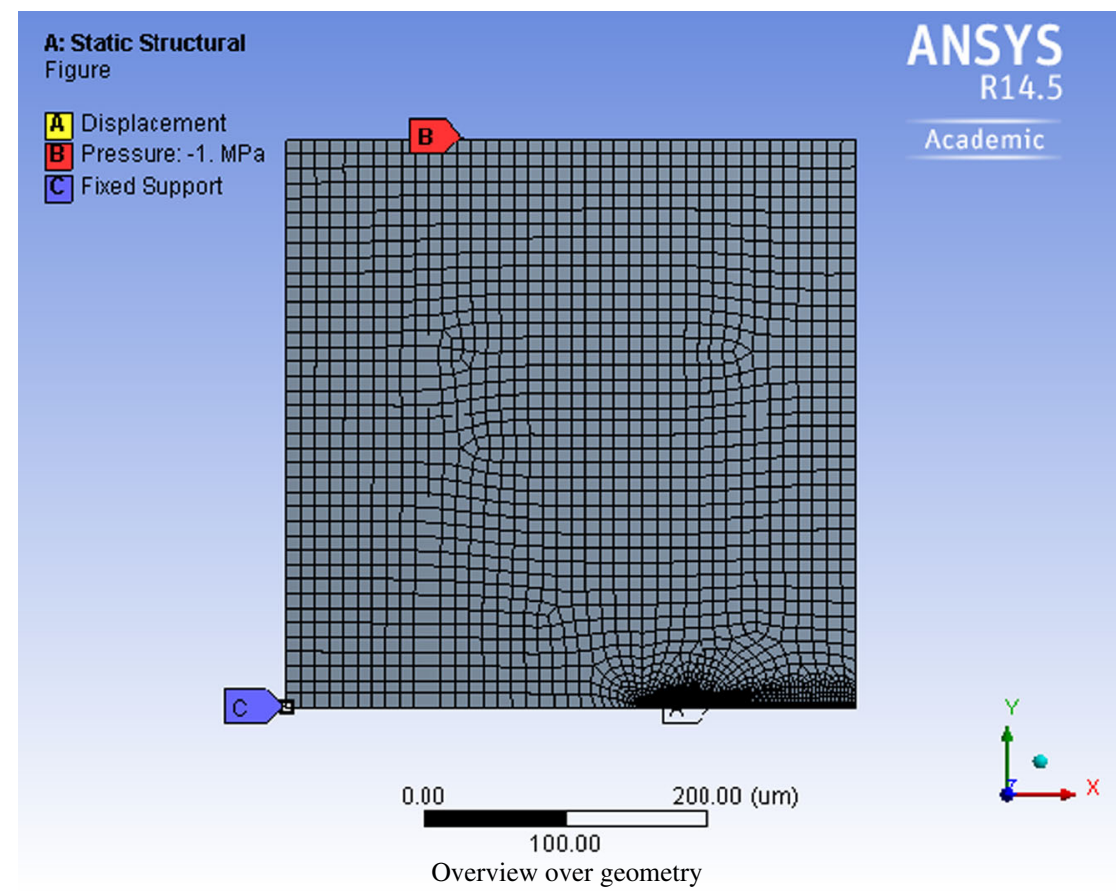

(b)

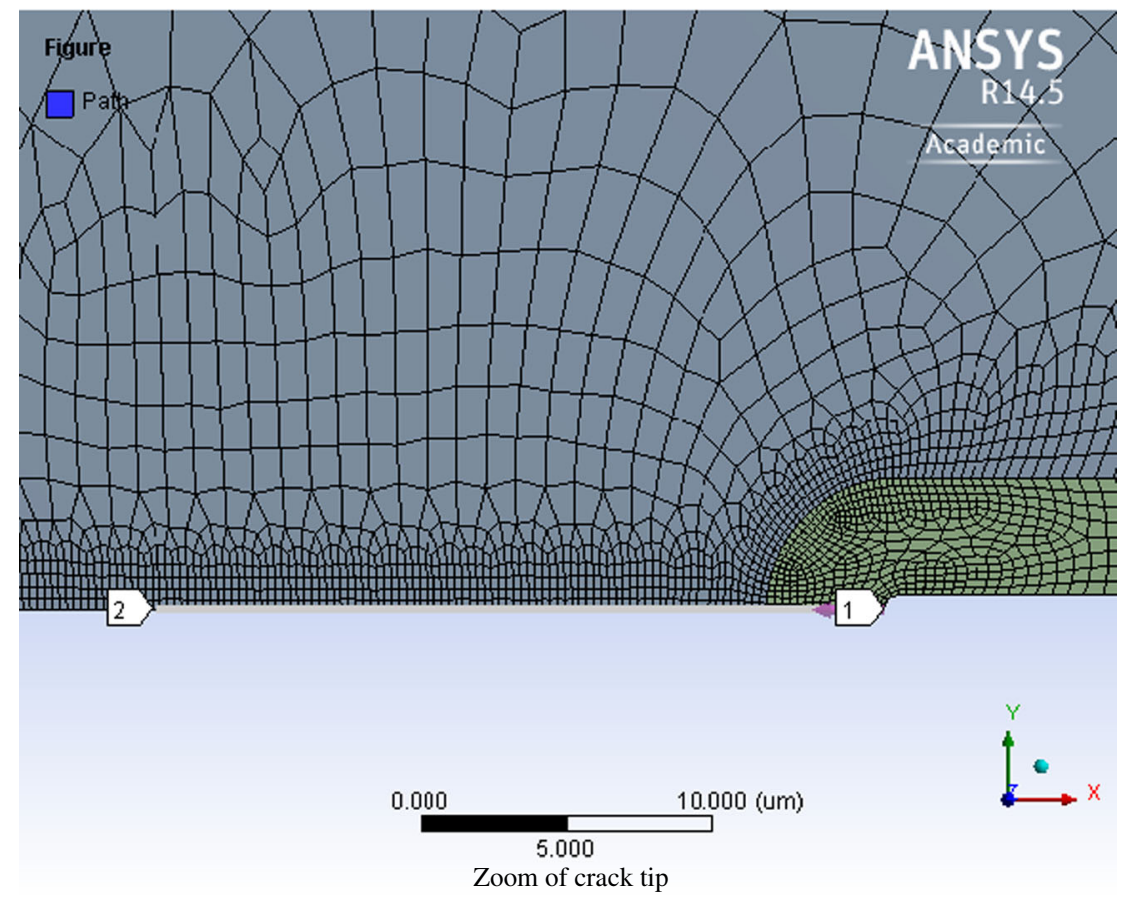

For the samples which were fractured in the quasi-static tensile test, then healed 90 min through SMA activation and tested the following day, slightly lower fatigue resistance was observed. For a load of $\Delta K=0.50 \mathrm{MPam}^{1 / 2}$, crack growth occurred and the sample was fractured rapidly after 13000 cycles. The latter sample almost instantly cracked by $1 \mathrm{~mm}$, then continued at a steady crack growth rate, stabilized for about 1000 cycles but finally crack growth resumed and rapidly changed to unstable crack growth, characterized by the steep slope before failure. For lower stress intensities, no crack progression was observed. 
Fig. 6 Equivalent von Mises stress distribution around the crack tip for the (a) pure epoxy case as well as the (b)

solvent-affected epoxy. Mind the different color map scales (a)

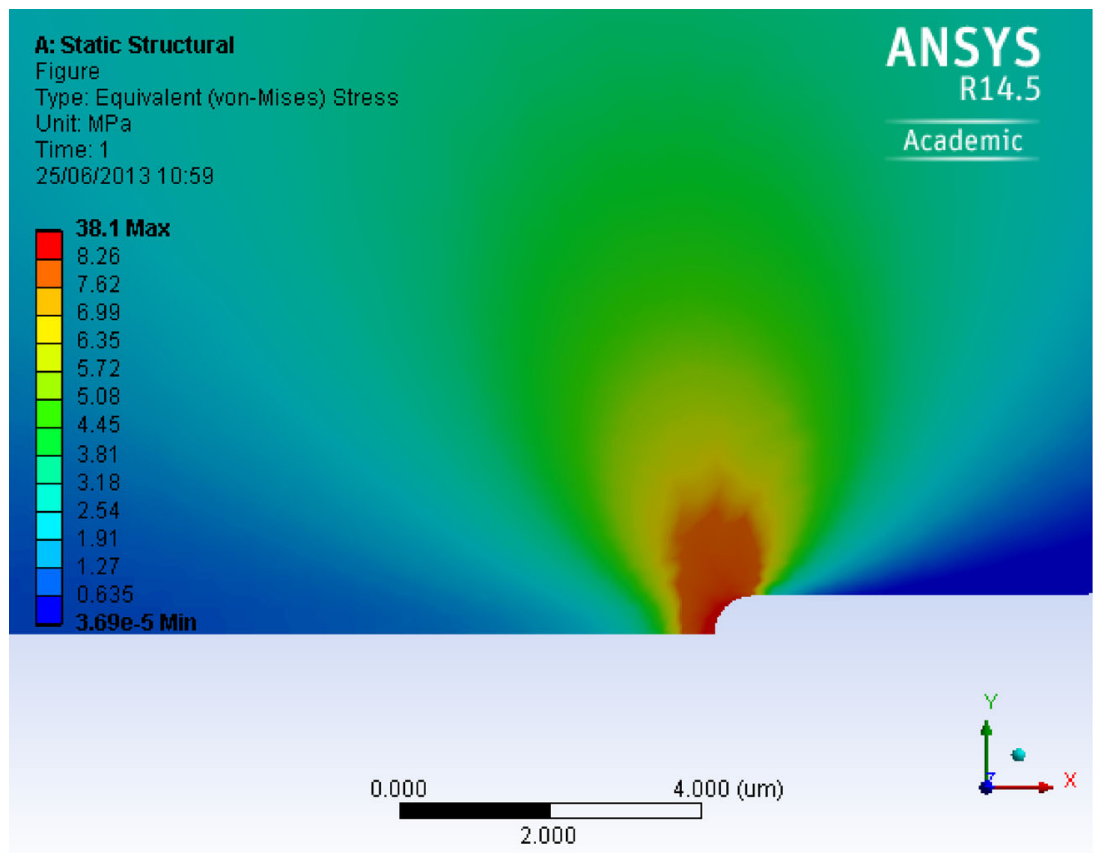

Stress in pure epoxy

(b)

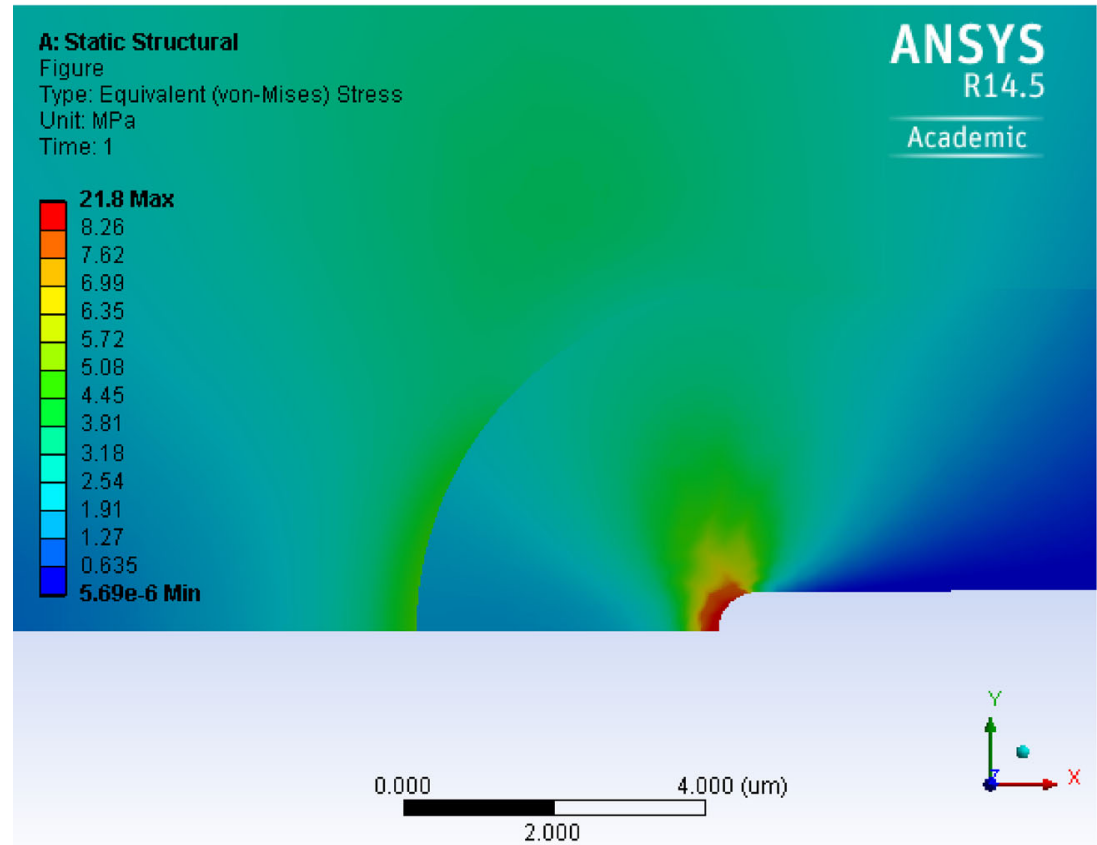

Stress in epoxy/solvent
Low-Cycle Tests with Rest Periods

After investigating continuous fatigue testing on virgin and healed samples, we also characterized the effect of rest periods during low-cycle fatigue tests. The tests were conducted on samples with or without SMA wires at a stress intensity of $\Delta K=0.50 \mathrm{MPam}^{1 / 2}$ and the rest periods were $10 \mathrm{~min}, 2 \mathrm{~h}$ and $24 \mathrm{~h}$. $10 \mathrm{~min}$ was chosen as a control as this duration is insufficient for significant bonding to take place. $2 \mathrm{~h}$ was chosen as this was in the order of the 
(a)

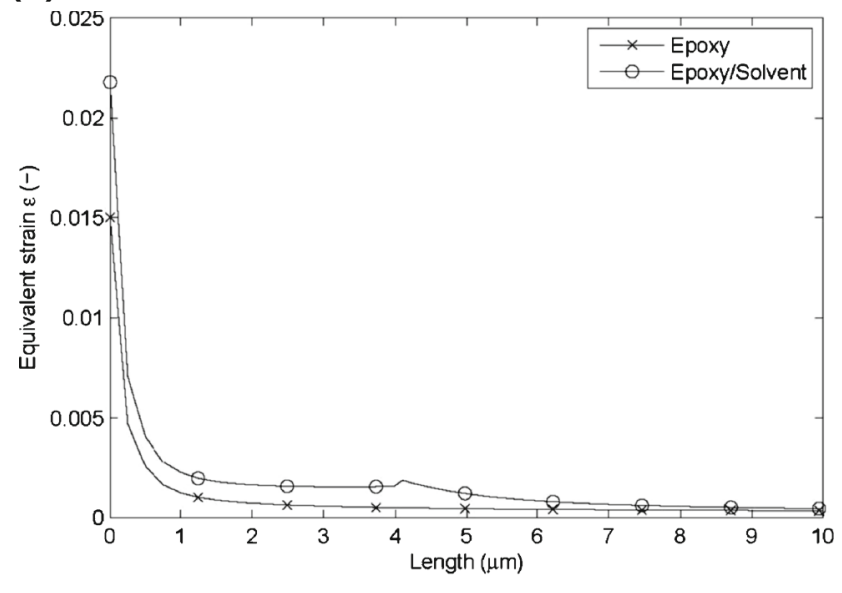

Strain for epoxy and epoxy/solvent

(b)

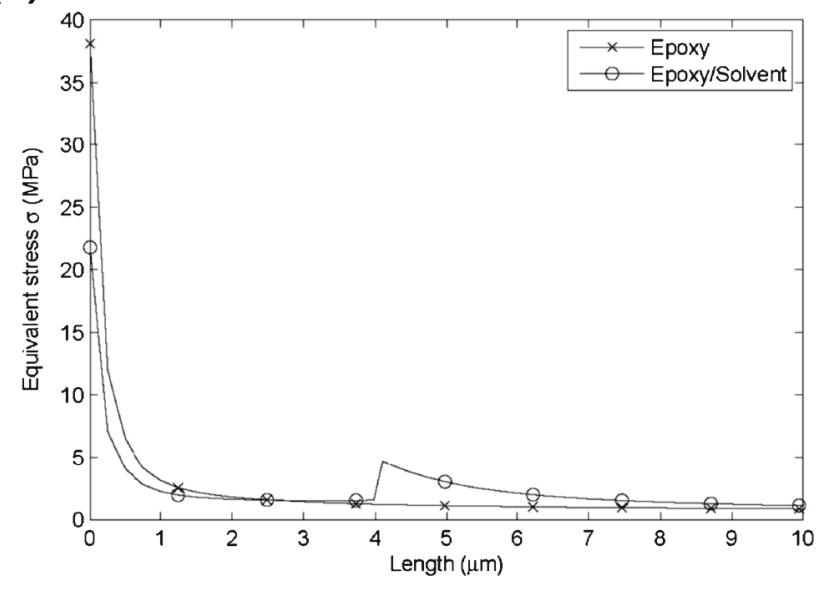

Stress for epoxy and epoxy/solvent

Fig. 7 (a) Strains and (b) stresses along the middle section as defined by the path shown in Fig. 5(b)

observed time for crack arrest. Finally $24 \mathrm{~h}$ is known to be sufficient time for maximal healing efficiency in static testing. In Fig. 9, representative results for $10 \mathrm{~min}$ and $2 \mathrm{~h}$ rest periods are shown. In all results discussed hereafter, the crack progression during the first 20000 cycles was at least $5 \mathrm{~mm}$.

After the 10 min rest period on samples without SMA wires, no significant effect on the overall trend of the crack progression was observed while with SMA activation, crack arrest was observed. Please note that after the rest period, a small jump (positive or negative) in crack length, in the order of 1-2 $\mathrm{mm}$, was often measured. The phenomenon is a data artifact due to the setup but it does not alter the interpretation of the results as the trends before and after the rest periods are considered.

For the $2 \mathrm{~h}$ rest period, crack arrest occurred with and without the activation of the SMA wires for these (a)

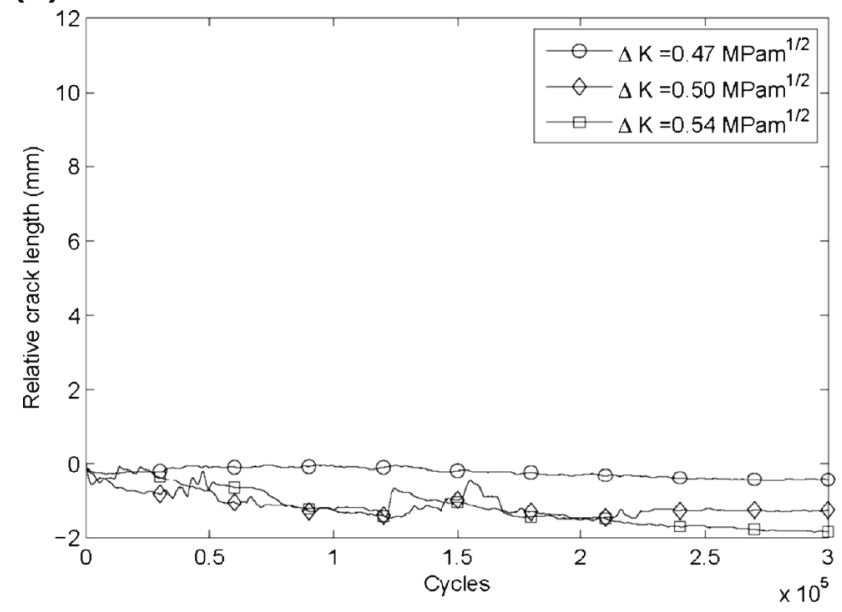

(b)

Virgin samples. No previous test

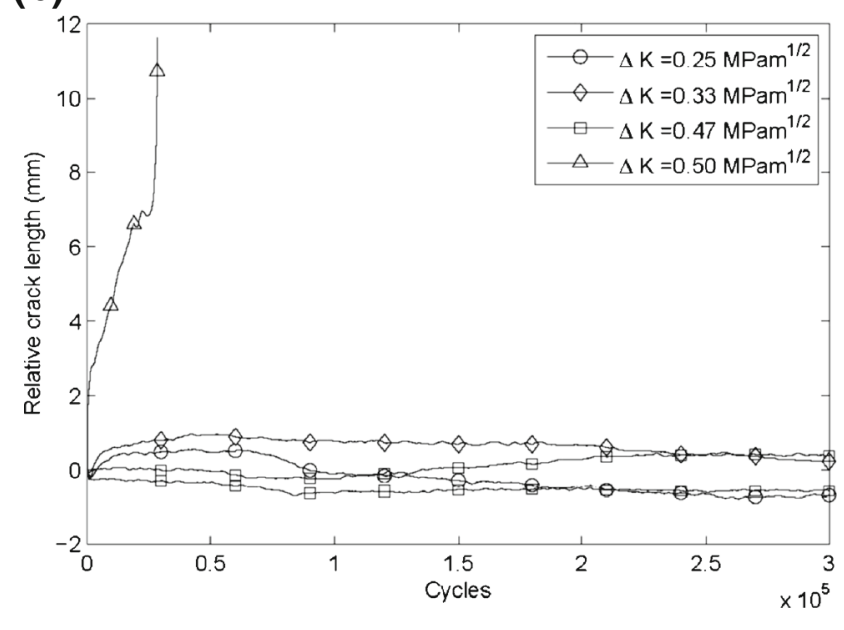

Healed samples. Fractured followed by SMA activation

Fig. 8 (a) Virgin and (b) healed TDCB samples loaded with $15 \mathrm{wt} \%$ EPA microcapsules and integrated SMA wires tested at different stress intensities without break

samples. In case of a $24 \mathrm{~h}$ rest period, a significant reduction of crack length was observed at the beginning of the test. The stiffening of the sample was caused by healing. However, crack progression was fast and the healed material broke within about 5000 cycles, after which the same trend as before the rest period was observed. If the SMA wires were activated for 90 min with a total rest period of $24 \mathrm{~h}$, the same behavior as found in samples that were statically broken, then healed through SMA wires was observed with no crack progression during fatigue testing after the rest period.

The results are summarized in Fig. 10. Per set of parameters, on average 5 samples were tested and clearly longer rest periods as well as SMA activation improve the fatigue response. 
(a)

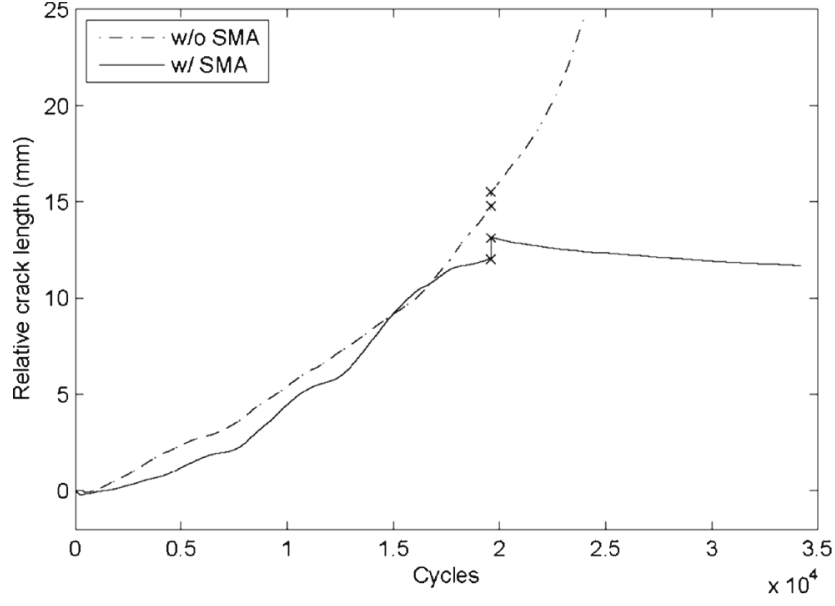

(b)

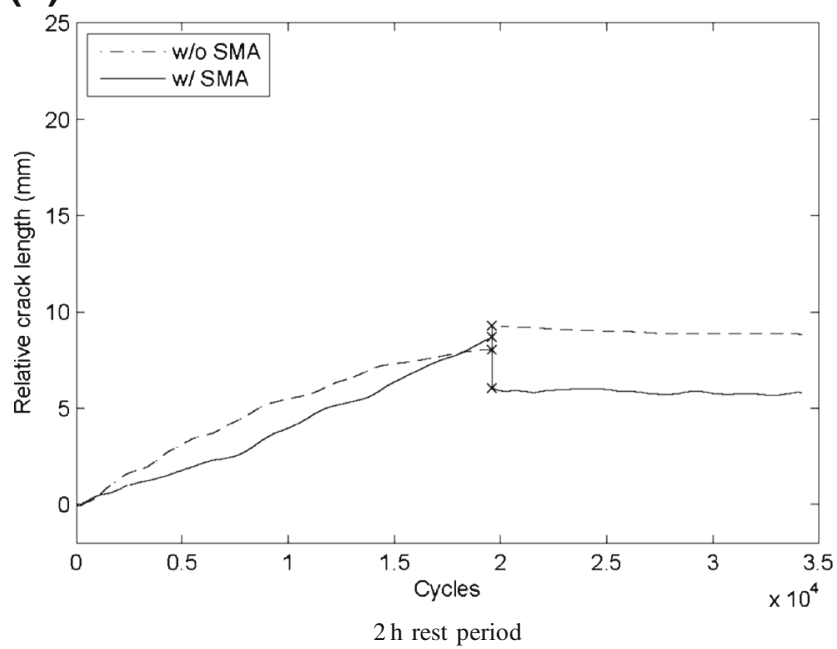

Fig. 9 Comparison of the effect of rest periods (marked with an " $x$ ") on low-cycle fatigue testing of PC1 epoxy. For (a) 10 min rest periods, no effect was observed unless the SMA wires were activated. For (b) $2 \mathrm{~h}$ rest periods, crack arrest was observed with or without SMA wire activation

\section{High Temperature Post-Cured Epoxy}

\section{Quasi-Static Testing}

The above findings are based on an under-cured resin, as was successfully demonstrated in static self-healing tests. Nonetheless, we showed in Section Fatigue Behavior of Epoxy Matrix with Embedded Self-Healing Agent that in the case of fatigue testing, enhanced fatigue life was observed even when continuously loading the samples, which inhibits crack face joining as is the case in static healing. In this section we therefore investigate the healing response of high-temperature cured epoxy.

First, we measured healing efficiency in static fracture toughness testing of this high-temperature cured epoxy

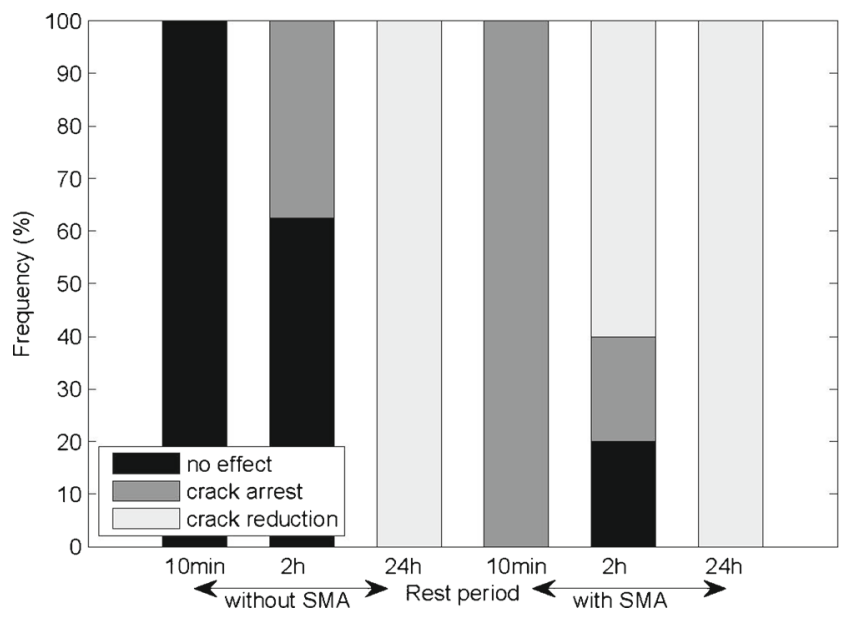

Fig. 10 Effect of rest period and SMA activation on crack propagation. The relative number of observed incidences of no effect/crack arrest/crack reduction are plotted against rest time and SMA activation

(PC2) and the results are found in Table 2. Unsurprisingly, the healed maximal force was very low for the PC2 epoxy as compared to the under-cured epoxy. The resulting healing efficiency dropped from $92.5 \%$ to $10.7 \%$ for the PC2 epoxy. The degree of cure for this PC2 epoxy was about $95 \%$ and therefore the residual monomer concentration is too low to effectively bond the fracture face.

Fatigue Testing

Next, we subjected PC2 epoxy samples with EPA microcapsules to different stress intensities for 300000 cycles. The results are found in Fig. 11. Only at a stress intensity of $\Delta K=0.35 \mathrm{MPam}^{1 / 2}$, very slow crack progression was measured. For higher stress intensities, samples showed stable crack growth followed by fast, unstable crack growth prior fracture. Compared to the previous results of PC1 epoxy, where fracture only occurred for $\Delta K=$ $0.56 \mathrm{MPam}^{1 / 2}$, we can conclude that the higher curing temperature not only inhibits static healing, but also inhibits the crack arrest phenomenon observed for PC1 epoxy.

This finding suggests that the higher degree of cure of the epoxy also completely inhibits the diffusion of the solvent into the matrix. Therefore we measured weight uptake

Table 2 Healing efficiency of PC1 \& PC2 samples during TDCB tests

\begin{tabular}{llcl}
\hline Post-cure & $F_{\max }[N]$ & $F_{\text {heal }}[\mathrm{N}]$ & Healing efficiency $[\%]$ \\
\hline PC1 & $81.9 \pm 13.0$ & $74.9 \pm 8.2$ & $92.5 \pm 11.20$ \\
PC2 & $87.5 \pm 7.1$ & $9.3 \pm 1.3$ & $10.7 \pm 2.0$ \\
\hline
\end{tabular}




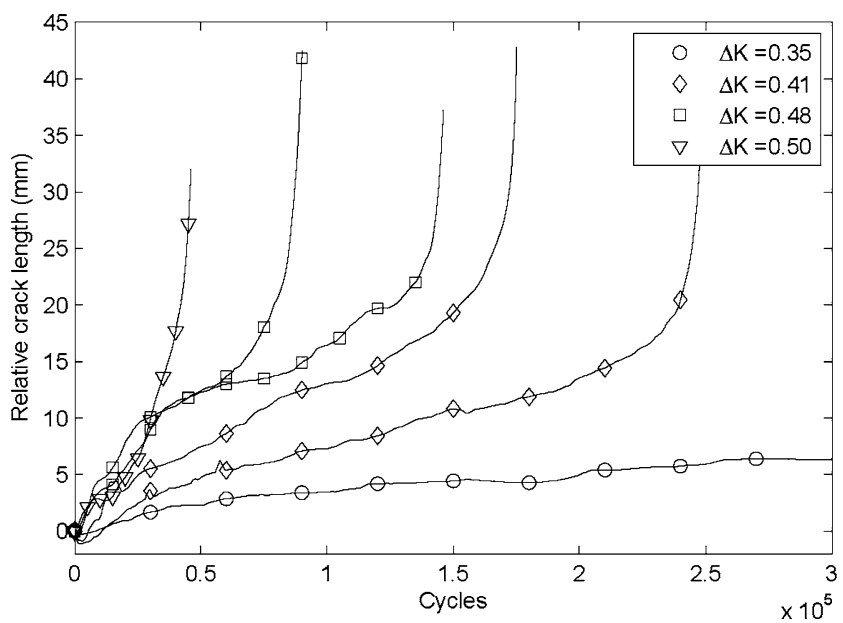

Fig. 11 Fatigue response of TDCB samples subjected to different stress intensities. Crack progression was higher as compared to under-cured epoxy for the same applied loads

of PC2 epoxy samples of $9.7 \mathrm{~mm}$ diameter and a height of $4.9 \mathrm{~mm}$ (ca. $0.42 \mathrm{~g}$ ) immersed in EPA solvent, as previously carried out for PC1 epoxy [20]. The results are averaged over at least 3 samples and reported in Fig. 12, along with our previous results for PC1 [20]. After 20 days, the PC1 epoxy had absorbed $4.50 \%$ while the PC2 epoxy increased in mass by $0.02 \%$. Almost no solvent uptake took place in the cured epoxy.

With respect to the discussed healing mechanisms of reaction of residual monomer and crack pinning through local plasticization, it is therefore clear that in fully cured samples, no sizable healing or crack retardation could be observed. This confirms the beneficial effect of solvent diffusion in PC1 samples.

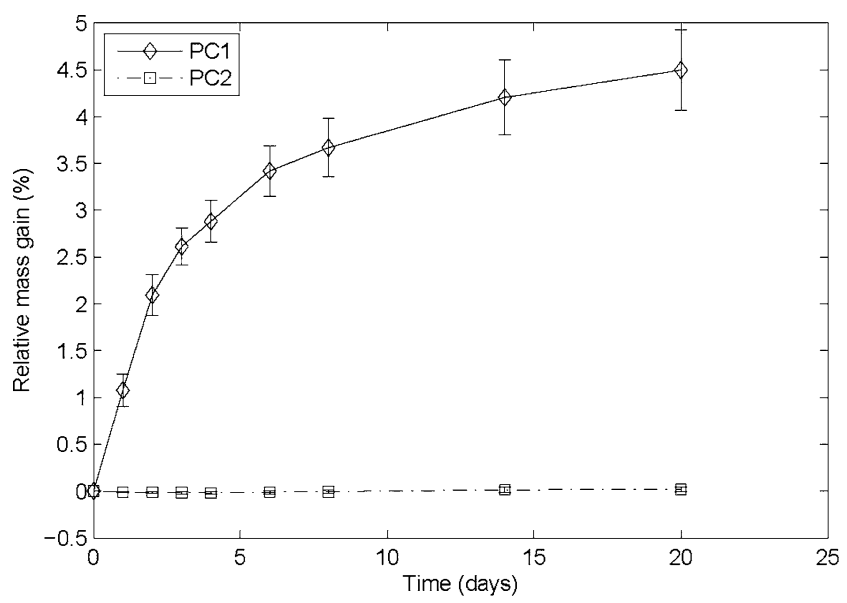

Fig. 12 Mass increase of under-cured (PC1) and almost fully cured (PC2) epoxy immersed in EPA solvent

\section{Conclusion}

A self-healing epoxy with EPA solvent-filled UF microcapsules and SMA wires was tested in fatigue. Complete crack arrest was observed for very high loading conditions and this phenomenon was attributed to the reduced stresses in the crack tip due to solvent diffusion. FEM modeling of the crack tip confirmed a decrease in stress when the epoxy matrix presented a lower modulus due to solvent-induced plasticization. Therefore, in an under-cured epoxy matrix with solvent-filled microcapsules, there exists a threshold stress intensity below which crack progression eventually stops while above, the diffusion rate cannot compete with the crack progression and a more classical behavior was observed. We also showed that SMA wires do help restore the fatigue life of a fully broken sample. If rest periods are respected, we showed that at high loading conditions, crack arrest was observed only if the rest duration was at least $2 \mathrm{~h}$. With SMA activation, already 10 min breaks were sufficient to arrest cracks at the same stress intensity. Finally, for a fully cured epoxy, we also showed that the solventbased healing system neither mends statically fractured crack faces, nor induces crack arrest during fatigue testing, though for different reasons. In the static case, both the low diffusion rate and the low residual monomer concentration are responsible for the very low healing efficiency, while in case of dynamic testing, the inhibition of solvent diffusion through the fully cured epoxy network impedes the crack arrest phenomenon observed in under-cured epoxy.

We therefore conclude that embedding solvent filled microcapsules into a glassy polymer matrix can have beneficial effects on fatigue resistance without relying on a self-healing effect. Rather the local diffusion and plasticization of the matrix are responsible for crack arrest in an otherwise brittle matrix.

Acknowledgments The authors gratefully acknowledge the financial support of the Swiss National Science Foundation (FNRS 511482), the Laboratory of Applied Mechanics and Reliability Analysis (LMAF) at EPFL for using their fatigue testing device and M. G. Sahini, E. Rotterman and S. Clerc for their technical assistance.

\section{References}

1. White SR, Sottos NR, Geubelle PH, Moore JS, Kessler MR, Sriram SR, Brown EN, Viswanathan S (2001) Autonomic healing of polymer composites. Nature 409(6822):794-797

2. Chen X, Dam MA, Ono K, Mal A, Shen H, Nutt SR, Sheran K, Wudl F (2002) A thermally re-mendable cross-linked polymeric material. Science 295(5560):1698-1702

3. Hayes SA, Jones FR, Marshiya K, Zhang W (2007) A self-healing thermosetting composite material. Compos A Appl Sci Manuf 38(4):1116-1120 
4. Bergman SD, Wudl F (2008) Mendable polymers. J Mater Chem 18(1):41-62

5. Berl V, Schmutz M, Krische MJ, Khoury RG, Lehn JM (2002) Supramolecular polymers generated from heterocomplementary monomers linked through multiple hydrogen-bonding arrays - formation, characterization, and properties. Chem Eur J 8(5):12271244

6. Kalista SJ, Ward TC, Oyetunji Z (2007) Self-healing of poly(ethylene-co-methacrylic acid) copolymers following projectile puncture. Mech Adv Mater Struct 14(5):391-397

7. Chen X, Wudl F, Mal AK, Shen H, Nutt SR (2003) New thermally remendable highly cross-linked polymeric materials. Macromolecules 36(6):1802-1807

8. Caruso MM, Delafuente DA, Ho V, Sottos NR, Moore JS, White SR (2007) Solvent-promoted self-healing epoxy materials. Macromolecules 40(25):8830-8832

9. Yan CY, Min ZR, Ming QZ, Chen J, Gui CY, Xue ML (2008) Selfhealing polymeric materials using epoxy/mercaptan as the healant. Macromolecules 41(14):5197-5202

10. Williams G, Trask R, Bond I (2007) A self-healing carbon fibre reinforced polymer for aerospace applications. Compos A Appl Sci Manuf 38(6):1525-1532

11. Toohey KS, Sottos NR, Lewis JA, Moore JS, White SR (2007) Self-healing materials with microvascular networks. Nat Mater 6(8):581-585

12. Williams HR, Trask RS, Weaver PM, Bond IP (2008) Minimum mass vascular networks in multifunctional materials. J R Soc Interface 5(18):55-65

13. Blaiszik BJ, Kramer SLB, Olugebefola SC, Moore JS, Sottos NR, White SR (2010) Self-healing polymers and composites. Annu Rev Mater Res 40(1):179-211

14. Kirkby E, de Oliveira R, Michaud V, Månson JA (2011) Impact localisation with fbg for a self-healing carbon fibre composite structure. Compos Struct 94(1):8-14

15. Kessler SS, Spearing SM, Soutis C (2002) Damage detection in composite materials using lamb wave methods. Smart Mater Struct 11(2):269-278

16. Kirkby EL, Rule JD, Michaud VJ, Sottos NR, White SR, Månson JAE (2008) Embedded shape-memory alloy wires for improved performance of self-healing polymers. Adv Funct Mater 18(15):2253-2260

17. Park JS, Takahashi K, Guo Z, Wang Y, Bolanos E, HamannSchaffner C, Murphy E, Wudl F, Hahn HT (2008) Towards development of a self-healing composite using a mendable polymer and resistive heating. J Compos Mater 42(26):2869-2881

18. van der Zwaag S, Schmets AJM (2007) Self healing materials : an alternative approach to 20 centuries of materials science. Springer, Dordrecht

19. Kirkby EL, Michaud VJ, Månson JAE, Sottos NR, White SR (2009) Performance of self-healing epoxy with microencapsulated healing agent and shape memory alloy wires. Polymer 50(23):5533-5538

20. Neuser S, Michaud V, White SR (2012) Improving solvent-based self-healing materials through shape memory alloys. Polymer 53(2):370-378

21. Yuan YC, Rong MZ, Zhang MQ, Yang GC (2009) Study of factors related to performance improvement of self-healing epoxy based on dual encapsulated healant. Polymer 50(24):57715781
22. Toohey KS, Hansen CJ, Lewis JA, White SR, Sottos NR (2009) Delivery of two-part self-healing chemistry via microvascular networks. Adv Funct Mater 19(9):1399-1405

23. Caruso MM, Blaiszik BJ, White SR, Sottos NR, Moore JS (2008) Full recovery of fracture toughness using a nontoxic solvent-based self-healing system. Adv Funct Mater 18(13):1898-1904

24. Weisenberger LA, Koenig JL (1990) Nmr imaging of diffusion processes in polymers: measurement of the spatial dependence of solvent mobility in partially swollen pmma rods. Macromolecules 23(9):2445-2453

25. Dutheillet Y, Mantle M, Vesely D, Gladden L (1999) Diffusion of water-acetic acid mixtures in epoxy. J Polym Sci B Polym Phys 37(23):3328-3336

26. Hamilton AR, Sottos NR, White SR (2012) Mitigation of fatigue damage in self-healing vascular materials. Polymer 53(24):55755581

27. Brown EN, White SR, Sottos NR (2005) Retardation and repair of fatigue cracks in a microcapsule toughened epoxy composite - part i: manual infiltration. Compos Sci Technol 65(15-16):2466-2473

28. Brown EN, White SR, Sottos NR (2005) Retardation and repair of fatigue cracks in a microcapsule toughened epoxy composite-part II: in situ self-healing. Compos Sci Technol 65(15-16):2474-2480

29. Brown EN, White SR, Sottos NR (2006) Fatigue crack propagation in microcapsule-toughened epoxy. J Mater Sci 41(19):62666273

30. Shin CS, Huang KC, Li RZ (1998) Artificial retardation of fatigue crack growth by the infiltration of cracks by foreign materials. Fatigue Fract Mater Struct 21(7):835-846

31. Caruso MM (2010) Solvent-based self-healing polymeric materials. PhD thesis, University of Illinois

32. Neuser S, Michaud V (2013) Effect of aging on the performance of solvent-based self-healing materials. Polym Chem. doi:10.1039/C3PY00064H

33. Blaiszik BJ, Caruso MM, McIlroy DA, Moore JS, White SR, Sottos NR (2009) Microcapsules filled with reactive solutions for self-healing materials. Polymer 50(4):990-997

34. Jones AS, Rule JD, Moore JS, White SR, Sottos NR (2006) Catalyst morphology and dissolution kinetics of self-healing polymers. Chem Mater 18(5):1312-1317

35. Rule JD, Brown EN, Sottos NR, White SR, Moore JS (2005) Wax-protected catalyst microspheres for efficient self-healing materials. Adv Mater 17(2):205-208

36. Beres W, Koul AK, Thamburaj R (1997) A tapered doublecantilever-beam specimen designed for constant-k testing at elevated temperatures. J Test Eval 25(6):536-542

37. Brown EN (2011) Use of the tapered double-cantilever beam geometry for fracture toughness measurements and its application to the quantification of self-healing. Journal Strain Anal Eng Des 46(3):167-186

38. Brown EN, Sottos NR, White SR (2002) Fracture testing of a selfhealing polymer composite. Exp Mech 42(4):372-379

39. Paris P, Erdogan F (1963) A critical analysis of crack propagation laws. J Basic Eng 85(4):528-534

40. Moy P, Karasz FE (1980) Epoxy-water interactions. Polym Eng Sci 20(4):315-319

41. Jones AS, Rule JD, Moore JS, Sottos NR, White SR (2007) Life extension of self-healing polymers with rapidly growing fatigue cracks. J R Soc Interface 4(13):395-403

42. ANSYS Inc http://www.ansys.com/. Accessed 7 Apr 2012 